\title{
Extramammary Paget's disease: A report of two cases and a review of literature
}

\section{Nidhi Gupta', Tushar Kanti Sarkar², Shashank Bhargava1}

\author{
${ }^{1}$ R. D. Gardi Medical College, Ujjain, MP, India, ${ }^{2}$ Paikpari RH, Kolaghat, Purba Medinipur, WB, India
}

Corresponding author: Dr. Shashank Bhargava, E-mail: shashank2811@gmail.com

\begin{abstract}
Extramammary Paget's disease (EMPD) is a rare, slow-growing intraepithelial adenocarcinoma in the anogenital and axillary regions of the body clinically mimicking inflammatory and infective diseases. Surgical excision is basically performed as a treatment for EMPD. Generally, the prognosis is poor. Herein, we report two cases of extramammary Paget's disease involving the vulval region in a 70-year-old female and the inguinal region along with the left part of the scrotum in a 57-year-old male. Neither had any underlying malignancy or metastasis.
\end{abstract}

Key words: Extra mammary Paget's disease; Vulva; Scrotum

\section{INTRODUCTION}

Mammary Paget's disease was first described by James Paget in 1874 [1]. It was in 1889 when Crocker described the first case of extramammary Paget's disease (EMPD), involving the scrotum and penis [2], and believed that the tumor derived from the sweat and sebaceous glands or hair follicles. EMPD mostly involves the vulva and anus, and is rarely found on the scrotum and penis [3]. In simple terms, it is an intraepithelial adenocarcinoma clinically presenting itself as a chronic eczema-like rash mainly in the external genital and axillary regions. EMPD is classified into primary (disease confined to the epidermis and dermis) and secondary (involvement of the visceral organs). It is most commonly mistaken for eczema or contact dermatitis. Other differential diagnoses to be considered are SCC, melanoma, and other benign papulosquamous diseases. In its early stages, EMPD is not invasive or metastatic, hence surgical excision gives satisfactory results. EMPD in its advanced progression is often difficult to treat and control, despite the availability of various therapeutic options, such as surgery, radiotherapy, and/or chemotherapy, including docetaxel and trastuzumab $[4,5]$.

\section{CASE REPORTS}

\section{Case 1}

A 70-year-old female presented herself to us with a mildly pruritic erosive lesion with crusting on the vulva persistent for the last two years (Fig. 1). In the past, the patient applied a topical steroid on the lesion but with no improvement in the size of the lesion. The lesion started as a reddish mildly pruritic macule to gradually become slightly scaly and eventually erode, which was further aggravated after the application of topical steroids. The patient was a known diabetic. The patient's medical history was unremarkable and she denied any history of malignancy. A physical examination revealed a healthy geriatric female without systemic symptoms. A histopathological examination of a biopsy specimen from the skin lesion revealed an acanthotic epidermis with suprabasal atypical cells present in clusters or singly migrating toward the superficial epidermal layer. These cells had pale cytoplasm with a high nuclearcytoplasmic ratio and prominent nuclei. Some of these cells showed a clear halo around the nucleus. The dermis showed dense lymphocytic infiltration (Fig. 2). Findings were consistent with EMPD of the vulva. 
Staging workup, which included chest radiography and computed tomography of the abdomen, revealed no features of metastasis. The patient underwent radiation therapy followed by vulvectomy with a 2 -cm margin to the macroscopic normal tissue and primary closure. Surgical margins were histopathologically negative and the wound healed without complications. A follow-up after two years revealed no signs of recurrence.

\section{Case 2}

A 57-year-old male presented himself with a ten-month history of an itchy, erythematous macule on the left groin, which was misdiagnosed as tinea cruris and was treated accordingly (Fig. 3). As the lesion failed to resolve, the diagnosis was changed to seborrheic dermatitis and the patient was treated with topical corticosteroids for several months. However, the lesion progressed to an erosive mass with extension to the left lateral scrotum. The medical history was unremarkable, except a history of transurethral resection of the prostate for benign prostatic hypertrophy without evidence of malignancy. Histopathology confirmed the diagnosis of EMPD. The patient was also evaluated for metastasis but with no evidence of it. The patient underwent a wide excision of the skin lesion without recurrence to date.

\section{DISCUSSION}

EMPD is a rare, slow-growing intraepithelial adenocarcinoma occurring in the age group of 50-80 years most commonly located on the vulva followed by the perianal region. Other rarer locations include the axilla, eyelids, scrotum, and penis. It shows a female preponderance, with a ratio of 1.4:1 [6-8]. Several theories have been proposed for the pathogenesis of EMPD. One theory suggests that the disease is the result of multiple foci of malignant transformation of a population of cells with a common embryological origin, while another theory suggests that it is the result of a metastasis of underlying malignant cells to the epidermis $[9,10]$. Some other investigators believe that Paget's cells are either derived from or differentiate toward exocrine and apocrine gland cells, thus supporting the view that EMPD is an intraepithelial metastasis of an underlying exocrine gland adenocarcinoma [8-10], which warrants a search for underlying malignancy. The patient may be asymptomatic or may show pruritus (most commonly), a burning sensation, or pain. The lesion is typically sharply demarcated and eczematous with crusting, scaling, and rarely ulceration. Clinically, EMPD is to be differentiated from fungal infections, contact dermatitis, SCC, LSC,

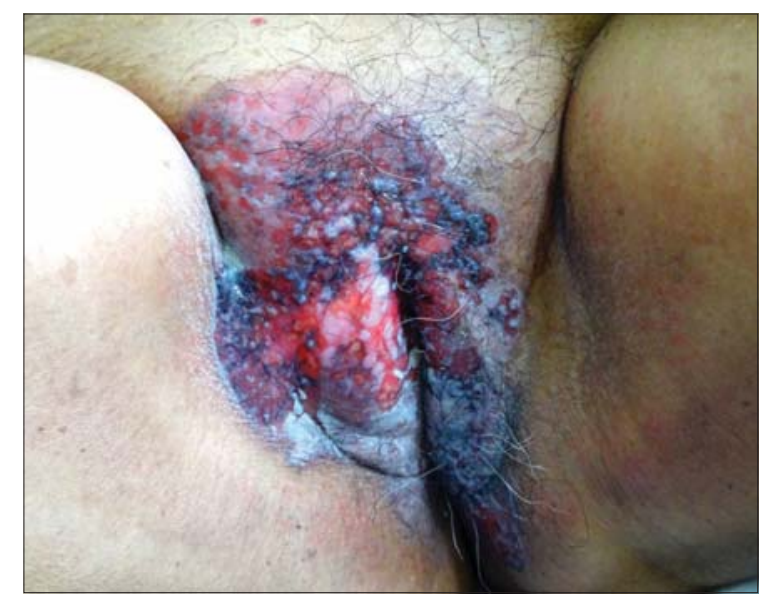

Figure 1: A mildly pruritic erosive lesion with crusting on the vulva in the first patient.

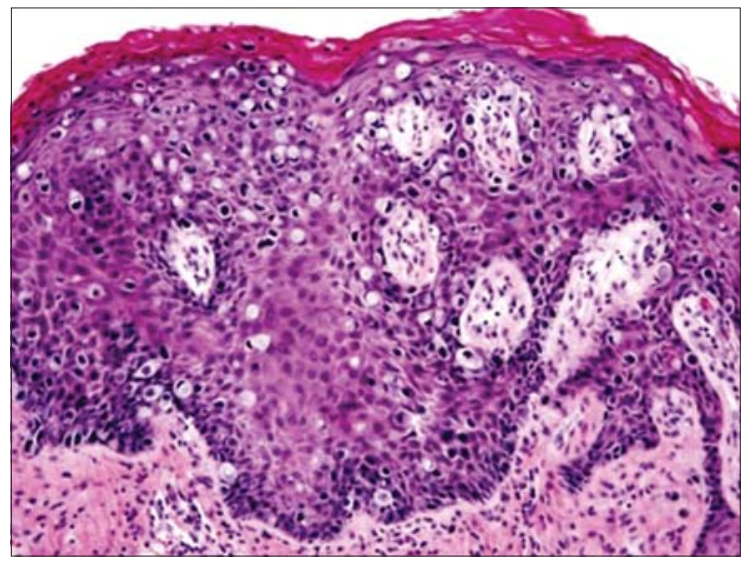

Figure 2: Histopathology showing an acanthotic epidermis with suprabasal atypical cells present in clusters or singly migrating toward the superficial epidermal layer; the cells with pale cytoplasm with a high nuclear-cytoplasmic ratio and prominent nuclei; some of the cells showing a clear halo around the nucleus.

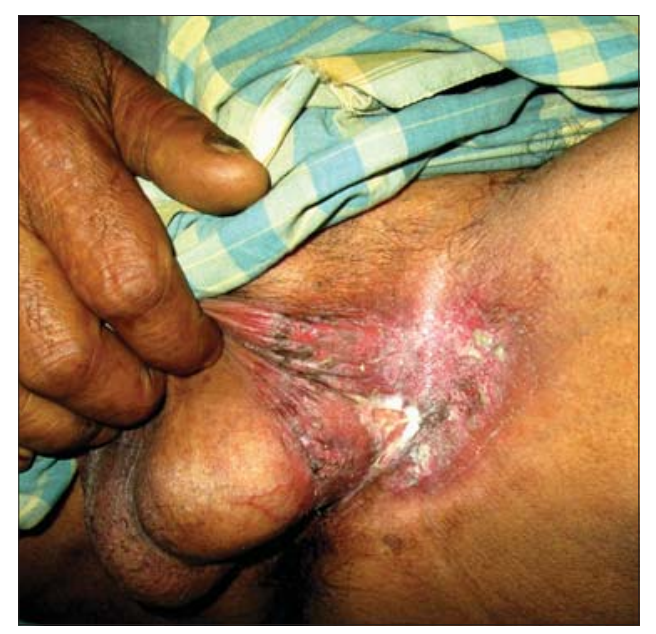

Figure 3: A mild itchy and erythematous plaque on the left groin in a 57-year-old male.

psoriasis, Bowen's disease, and melanoma $[6,7,9,10]$. However, failure to treatment gravitates toward the 
diagnosis of EMPD. Histologically, the presence of Paget's cells (large vacuolated cells containing basophilic or amphophilic finely granular cytoplasm, a large nucleus with atypia, and a prominent nucleolus nucleus) in the epidermis and/or adnexal epithelium confirms the diagnosis. The Paget's cells may be dispersed singly or form clusters, glandular structures, or a solid nest. In most of the cases (more than 90\%), tumor cells contain cytoplasmic mucin, staining positively with mucicarmine and a periodic acid-Schiff reagent [11]. A few cases may show the presence of melanin granules [12], which may be due to: 1) chemotactic factors produced by the neoplastic cells, generating a proliferation of dendritic melanocytes; and 2) Paget's cells phagocytosing melanin from the melanocytes. These cases mimic melanoma both clinically and histologically. However, in EMPD, the atypical cells with melanin are situated in the suprabasal layer, whereas, in melanomas, the malignant cells usually also surround the dermoepidermal junction [13]. The diagnosis may further be confirmed by immunohistochemistry, being positive for CK7, EMA, and CEA. Paget's cells express HER2/neu receptors and c-erb-2 oncogene, indicating a biological origin similar to that of breast carcinoma [14]. EMPD also has a high risk of noncontiguous malignancy. $24 \%$ of patients with EMPD of the penis, scrotum, or groin develop malignancy of the genitourinary tract, including prostate, bladder, and rectal carcinoma [15], while vulval EMPD has concomitant urogenital malignancies in 15\% of patients. Also, $33 \%$ of cases of perianal EMPD may develop colorectal adenocarcinoma. Therefore, a routine study for carcinoembryonic antigen and low molecular weight cytokeratin is to be done if secondary EMPD and internal adenocarcinoma are suspected. Since prognosis for localized EMPD is better than that for the invasive form of the disease, the treatment differs. Noninvasive EMPD can be treated with a wide local excision with a large margin, Mohs micrographic surgery (lesser recurrence), laser therapy, radiation, topical imiquimod, photodynamic therapy, and CO2 laser vaporization [7]. Lymph node dissection is performed only if there is clinical evidence of involvement. Invasive EMPD requires adjuvant therapy, such as radiotherapy or systemic chemotherapy. Chemoradiotherapy with 5-fluorouracil and mitomycin-C is effective. Also, monthly or weekly therapy with docetaxel and trastuzumab has proven to be effective.

\section{CONCLUSION}

EMPD must always be kept in mind as a differential diagnosis if a dermatitis involving sites such as the vulva or scrotum fails to respond to conventional treatment options. A prompt skin biopsy will help to reach a diagnosis for correct and timely management of EMPD.

\section{Consent}

The examination of the patient was conducted according to the principles of the Declaration of Helsinki.

The authors certify that they have obtained all appropriate patient consent forms, in which the patients gave their consent for images and other clinical information to be included in the journal. The patients understand that their names and initials will not be published and due effort will be made to conceal their identity, but that anonymity cannot be guaranteed.

\section{REFERENCES}

1. Paget J. On disease of the mammary areola preceding cancer of the mammary gland. St Bartholemew Hospital Res London. 1874;10:87-9.

2. Crocker HR. Paget's disease affecting the scrotum and penis. Transact Pathol Soc London. 1888-1889;40:187-91.

3. Juang GD, Lin MY, Hwang TI. Extramammary Paget's disease of the scrotum. J Chinese Med Assoc. 2011;74;325-8.

4. Yoshihara Y, Itoh M, Nobeyama Y, Nakagawa H. A case of advanced extramammary Paget's disease successfully controlled by monthly but not weekly Docetaxel chemotherapy. Case Rep Oncol. 2016;9:6-10.

5. Zhang X, Jin W, Zhu H, Yu H. Extramammary Paget's disease in Two Brothers. Indian J Dermatol. 2015;60:423.

6. Diatta BA, Hind H, Seck NB, Diadie S, Deh A, Diop K, et al. [Etiologic profi le of breast dermatoses in Dakar: A prospective study on 125 cases]. Our Dermatol Online. 2019;10:244-50.

7. Qian YT, Ma DL. Extramammary Paget disease of the glans penis. CMAJ. 2018;190:E1142.

8. Zhao Y, Gong X, Li N, Zhu Q, Yu D, Jin X. Primary extramammary Paget's disease: a clinicopathological study of 28 cases. Int J Clin Exp Pathol. 2019;12:3426-32.

9. Yamamoto T. Epidermotropic pagetoid spread and squamous cell carcinoma in situ in the overlying epidermis of Merkel cell carcinoma. Our Dermatol Online. 2014;5:210-1.

10. Li L, Wang C, Wei M, Huang Q, Wei L, Pan N, et al. Mohs micrographic surgery combined local flaps in treatment of EMPD: A retrospective case. Dermatol Ther. 2018;31:e12663.

11. Marcoval J, Penín RM, Vidal A, Bermejo J. Extramammary Paget Disease. Enfermedad de Paget extramamaria. Actas Dermosifiliogr. 2020;11:306-12.

12. Law C, Eykman E, Pennington T, Ip J, Pincott S. Perianal extramammary Paget's disease: a diagnosis worth considering. ANZ J Surg. 2020;10.1111/ans.15933.

13. Lydrup E, Berg JO, Hjorth SV, Hølmich LR, Lock-Andersen J, Chakera AH. Extramammary Paget's disease and Melanoma: 2 cases of double cancers. Case Rep Dermatol. 2020;12:107-13.

14. Vani BR, Thejaswini MU, Srinivasamurthy V, Rao MS. Pigmented Paget's disease of nipple: a diagnostic challenge on cytology. J Cytol. 2013;30:68-70.

15. Choi SL, Park SB, Lee ES, Park HJ, Lee JB, Choi BI. Imaging features of extramammary Paget disease of the scrotum: A case report. Ultrasound Q. 2020;36:84-6.

Copyright by Nidhi Gupta, et al. This is an open-access article distributed under the terms of the Creative Commons Attribution License, which permits unrestricted use, distribution, and reproduction in any medium, provided the original author and source are credited.

Source of Support: Nil, Conflict of Interest: None declared. 